\title{
Analysis
}

\section{Transgender health care in primary care}

\section{INTRODUCTION}

'The expression of gender characteristics, including identities, thatarenotstereotypically associated with one's assigned sex at birth is a common and culturally diverse human phenomenon (that) should not be judged as inherently pathological or negative."

Recently the World Health Organization has moved to re-define gender identityrelated health and now uses the umbrella term transgender to describe 'a diverse group of people whose internal sense of gender is different than that which they were assigned at birth.?

This term is growing in familiarity globally and does not imply a medical condition. The term includes people living in accordance with their gender identity in the absence of medical treatment and those undergoing gender-affirmative health care to support and affirm their gender identity including those with non-binary gender identities. ${ }^{2}$

\section{PREVALENCE}

Increasing numbers of people are either gender questioning or identifying as transgender. Accurate epidemiological data are not available on how many people globally identify as gender questioning or transgender but there is evidence that the number of patients accessing gender-affirming health services is increasing. ${ }^{2}$ This is particularly true for children and adolescents. ${ }^{3}$

Recent studies suggest that the prevalence of a self-reported transgender identity in children, adolescents, and adults ranges from $0.3 \%$ to $2.7 \%$, significantly higher than prevalence estimates based on adult clinic-based referrals. ${ }^{2,3}$ Furthermore, an expanded definition of gender variance results in higher prevalence estimates. ${ }^{3}$ However, cultural norms around gender expression may impact on self-report and most of the reported prevalence studies have been conducted in European countries. ${ }^{1,3}$

\section{TRANSGENDER HEALTHCARE NEEDS}

There is an increasing focus on the healthcare needs of transgender people. ${ }^{1,2,4}$ This patient group and their families have specific medical, social, and psychological needs that have not been adequately met by routine healthcare services including primary care. ${ }^{2,4}$

Transgender people experience high levels of discrimination, stigma, social exclusion, and harassment in many aspects of their

\section{GPs play a critical role in providing a safe space for gender-questioning and transgender people lincluding childrenl to disclose their issues and get access to appropriate support and referral pathways.}

lives including in healthcare settings. ${ }^{2}$ Access to specialist gender-affirming services is challenging with long waiting lists and lack of services in many regions. ${ }^{5}$ Many fear disclosing gender identity to their healthcare providers including their GPs, which may have a negative impact on healthcare provision. ${ }^{6}$

Compared with their cis-gender peers, transgender people experience many health disparities including higher rates of HIV infection, sexually transmitted infections, substance use, mental illness, and suicide. 2,7 Delays in accessing essential health-related support to enable transitioning can exacerbate these risks. ${ }^{4}$

Studies have shown that healthcare providers struggle to provide appropriate and sensitive gender-affirming care to this group including the use of acceptable terminology. This deficit is often linked to inadequate knowledge and training of healthcare providers. ${ }^{6,8}$

There is also a recognised deficit of quality research on transgender issues that impacts negatively on our understanding of their healthcare needs.

\section{IMPROVING TRANSGENDER HEALTH CARE IN PRIMARY CARE}

The General Medical Council and GP professional bodies in Ireland and the UK have published guidance and training modules on transgender health. $4,5,9,10$ However, many challenges remain in how to provide transgender people with timely and quality primary health care appropriate to their needs.

GPs play a critical role in providing a safe space for gender-questioning and transgender people (including children) to disclose their issues and get access to appropriate support and referral pathways. ${ }^{4,6}$ GPs are invaluable support to family members and other support networks who may struggle to understand gender identity and the appropriate terms to use. With a sensitive and non-judgemental approach to healthcare provision, GPs can help reduce the existing health inequities between this minority group and the rest of the population. ${ }^{8}$ Reassuring patients about confidentiality is also important. 4,6

It is important that GPs and their staff are aware of the correct terminology to use, and that we seek and clarify with the patient their name, pronoun, and preferred anatomical terms. ${ }^{4,8}$ These should be documented in the patient's medical records with all practice staff aware of how to address the patient. Providing non-binary and free-text options on practice registration forms will send a message of acceptance.

It is also important that GPs know the basics of gender-affirming health care and are aware of referral pathways for support and specialist treatment services. ${ }^{1,4,8}$

While the management of routine health care for transgender people is similar to their cis-gender peers, there are some issues that are unique to transgender healthcare delivery. ${ }^{1,4}$ Some of these can be very sensitive and how we approach them will gain a sense of trust with our patients. ${ }^{4.8}$

These issues include:

- physical examination, in particular chest and genital examination;

- appropriate inclusion in national cervical and breast screening programmes:

- prostate exams in trans women

- fertility and pregnancy care;

- sexually transmitted infection (STI) screening;

- management of cancer or genetic predisposition syndromes;

- patient may source hormone therapy over the internet and present for care around its management lincluding when their source may be abruptly stopped);

- patients may present for pre- or postgender-affirming operative care; and

- the use of puberty blockers in adolescents. 


\section{EXPANDING THE ROLE OF PRIMARY CARE IN TRANSGENDER HEALTH CARE}

A number of transgender people will embark on social or medical transitions. In most jurisdictions, specialist services are hard to access, costly, and the time delay for patients can be particularly stressful. 5

There are conflicting views on the role of primary care in providing transgender health care. Some GPs believe that most transgender health concerns can, and should, be managed in primary care. ${ }^{8}$ The care of these patients is not simply limited to hormone treatment and surgery, but incorporates an appreciation of wider psychosocial and professional issues. The 'whole-person' approach of primary care makes it a good fit for addressing the health needs of transgender people. ${ }^{1,4}$

However, there are concerns about the level of competency needed to provide some aspects of the medical care that may be required (for example, hormone therapy, preand post-gender-affirming surgery care, and puberty blockers). ${ }^{5}$ Given the deficits in the availability of specialist services, many GPs are concerned that they may be left to fill this void without the necessary terminology, training, and skill set. ${ }^{5}$

Both the Royal and Irish College of General Practitioners recognise the important role that GPs play in caring for transgender patients. ${ }^{4,5}$ Both colleges identify lack of training and the timely access to specialist services as major barriers to GP involvement in transgender health care and advocate for an urgent increase in specialist capacity and increased training needs for GPs.,5

However, this existing position does not address the present needs of many transgender patients. Many are still accessing hormone therapies online and suffer great stress in not being able to access gender-affirming surgery options in a timely manner. There may be a need for a more blended approach to treatment provision with some GPs developing a special interest in the field and providing support to their primary care colleagues. This approach has been successfully developed by the Indigo project in Manchester."

This approach could be augmented by the remote access to specialist service. Primary care has already developed a similar model for community hepatitis C treatment and more recently in the remote management of COVID-19.12

\section{MEDICAL EDUCATION}

There is an identified deficit in the amount of LGBT training in medical curricula, which impacts on the confidence and competence of doctors to provide gender-affirming care. $6,8,13$
It is recognised that educating healthcare providers about LGBT-related health is the most effective way to improve healthcare delivery to this population. ${ }^{6.13}$ It is a priority to incorporate more LGBT training into both undergraduate and postgraduate medical GP training and professional development. This may challenge some GPs to question their cis-normative and heteronormative assumptions that can negatively impact the care of their LGBT patients.

\section{CONCLUSION}

The care of transgender and genderquestioning people in primary care is of increasing relevance. It requires an open and non-judgemental approach, correct use of terminology, and knowledge of treatment and support services. Increasing specialist training on LGBT is critical to provide GPs with the skills and knowledge to provide appropriate care to this vulnerable group who suffer disproportionate levels of physical and mental health issues compared with their cis-gender peers. There is also an opportunity to review how specialist transgender treatment services are provided including the options of developing the role of GPs with a special interest and the use of remote models for specialist transgender healthcare delivery. Increased funding and research in the area is a priority.

\section{REFERENCES}

1. Coleman E, Bockting W, Botzer M, et al. Standards of care for the health of transsexual, transgender, and gender nonconforming people. 2012. https://www. wpath.org/publications/soc laccessed 30 Jun 2021)

2. World Health Organization Regional Office for Europe. WHO/Europe brief - transgender health in the context of ICD-11. https:// www.euro.who.int/en/health-topics/healthdeterminants/gender/gender-definitions/ whoeurope-brief-transgender-health-in-thecontext-of-icd-11 laccessed 30 Jun 2021).

3. Zhang Q, Goodman M, Adams N, et al. Epidemiological considerations in transgender health: a systematic review with focus on higher quality data. Int $J$ Transgender Heal 2020; 21(2): 125-137.

4. Crowley D, Lacey V, Quality and Safety in Practice Committee. Guide for providing care for transgender patients in primary care. Quick reference guide v.2. Dublin: Irish College of General Practitioners, 2021.

5. Royal College of General Practitioners. The role of the GP in caring for gender-questioning and transgender patients: RCGP position statement. 2019. https://www.rcgp.org.uk/-/ media/Files/Policy/A-Z-policy/2019/RCGP position-statement-providing-care-for-gendertransgender-patients-june-2019.ashx laccessed 30 Jun 2021).

6. Brooks H, Llewellyn CD, Nadarzynski T, et al

\section{ADDRESS FOR CORRESPONDENCE}

Des Crowley

School of Medicine, University College Dublin,

Belfield, Dublin 4, Ireland.

\section{Email: doctordesahotmail.com}

\section{Des Crowley,}

Associate Professor, School of Medicine, University College Dublin, Dublin, Ireland; Irish College of General Practitioners, Dublin, Ireland.

\section{Walter Cullen,}

Professor of Urban General Practice, School of Medicine, University College Dublin, Dublin, Ireland: Irish College of General Practitioners, Dublin, Ireland.

\section{Marie Claire Van Hout,}

Professor of Public Health Policy and Practice, Public Health Institute, John Moores University, Liverpool, UK.

\section{Provenance}

Freely submitted; externally peer reviewed.

\section{Competing interests}

The authors have declared no competing interests.

DOI: https://doi.org/10.3399/bjgp21X716753

Sexual orientation disclosure in health care: a systematic review. Br J Gen Pract 2018; DOI: https://doi.org/10.3399/bjgp18X694841.

7. Reisner SL, Poteat T, Keatley J, et al. Global health burden and needs of transgender populations: a review. Lancet 2016; 388(10042): 412-436

8. Hashemi L, Weinreb J, Weimer AK, Weiss RL. Transgender care in the primary care setting: a review of guidelines and literature. Fed Pract 2018; 35(7): 30-37.

9. General Medical Council. Trans healthcare https://www.gmc-uk.org/ethical-guidance/ ethical-hub/trans-healthcare laccessed 30 Jun 2021).

10. Royal College of General Practitioners. LGBT health hub. 2021. https://elearning.rcgp.org.uk/ $\mathrm{mod} /$ page/view.php?id=9380 laccessed 30 Jun 2021).

11. Indigo Gender Service. About us. https:// indigogenderservice.uk/about-us laccessed 30 Jun 2021)

12. Ní Cheallaigh $C, O^{\prime}$ Leary $A$, Keating $S$, et al. Telementoring with project $\mathrm{ECHO}$ : a pilot study in Europe. BMJ Innov 2017; 3(3): 144151.

13. Arthur S, Jamieson A, Cross H, et al. Medical students' awareness of health issues, attitudes, and confidence about caring for lesbian, gay, bisexual and transgender patients: a cross-sectional survey. BMC Med Educ 2021; 21(1): 56 . 